\title{
The Impact of Community Health Coalitions On Preventative Health Outcomes: A Systematic Review
}

\author{
Emily Shockley ${ }^{1}$, Aliya Amin ${ }^{2,3}$, Dennis Savaiano ${ }^{2,4}$ \\ ${ }^{1}$ Indiana University School of Medicine, ${ }^{2}$ Indiana Clinical and Translational Sciences Institute \\ Community Health Partnerships, ${ }^{3}$ Cardiovascular and Diabetes Coalition of Indiana, ${ }^{4}$ Purdue \\ University Department of Nutrition Science
}

Background/Objective: Six out of ten deaths in the United States are caused by chronic diseases. There are multiple approaches to prevention and/or early detection of chronic diseases through screenings, nutrition, physical activity, and more. Unfortunately, a large portion of the US population have low rates of preventative care. Community health coalitions have the potential to improve preventative health outcomes by partnering with key members of communities to design programs that best fit community needs. In this systematic review, we examine evidence that community health coalitions can improve preventative health outcomes.

Methods: PubMed, WebofScience, CINAHL, and PsycINFO were searched for (outcome OR health outcome) AND (prevention) AND (diabetes OR hypertension OR cancer OR prenatal) AND (community-based participatory research OR community coalition OR coalition OR health coalition). After screening 490 articles, 30 full-text, peer-reviewed, English articles meeting inclusion criteria of health coalition, prevention, health outcomes reported, and conducted in the United States were reviewed.

Results: 27 of the 30 studies showed community health coalitions having a positive impact. Roughly half of the articles were randomized control trials. Community members and academia were represented in $80 \%$ and $77 \%$, respectively, of the coalitions described, while other representations included faith-based organizations, health care, public health, industry, and education. Diabetes and cancer represented $74 \%$ of the targeted preventions addressed. $90 \%$ of the interventions focused on educating the community, and $33 \%$ focused on increasing health screening rates. $60 \%$ utilized trained community members to deliver interventions. $60 \%$ reported health measurements, $33 \%$ reported screening rates, and $30 \%$ reported knowledge and awareness.

Conclusion, Impact, \& Implications: While the evidence suggests community health coalitions are effective in improving preventative health outcomes, the quality of the studies remain low. There is a need for increased rigor of study design and assessment in order to determine the true efficacy of community health coalitions. The majority of the randomized-control trials in this review were rated as low-quality, highlighting the difficulty of designing a randomized, controlled study in a community. Inadequate funding, lack of rigorous design, or ethical challenges can limit the quality of the assessment. Further, although prenatal care was included as a search term, there were no coalitions found that addressed this type of preventative care. This points to a future need for the creation of or increased publications from prenatal health coalitions. 